\title{
Efficient inhibition of ovarian cancer by recombinant CXC chemokine ligand 10 delivered by novel biodegradable cationic heparin-polyethyleneimine nanogels
}

\author{
FAN YANG, MALIN GOU, HONGXIN DENG, TAO YI, QIAN ZHONG, YUQUAN WEI and XIA ZHAO \\ Department of Gynecology and Obstetrics, Key Laboratory of Obstetric and Gynecologic and Pediatric \\ Diseases and Birth Defects of Ministry of Education, West China Second Hospital; State Key Laboratory \\ of Biotherapy and Cancer Center, Sichuan University, Chengdu 610041, Sichuan, P.R. China
}

Received March 6, 2012; Accepted May 9, 2012

DOI: $10.3892 /$ or.2012.1853

\begin{abstract}
Currently, great interest is focused on the antineoplastic effects of CXC chemokine ligand 10 (IP-10/CXCL10). IP-10 has shown significant antitumor and anti-metastatic properties via immunological, antiangiogenic and anti-neoplastic mechanisms. However, very few studies on the antitumor activity of IP-10 in human ovarian cancer have been reported. The use of polymeric nanoparticles to deliver functional genes intraperitoneally holds much promise as an effective therapy for ovarian cancer. In our study, a recombinant plasmid expressing IP-10 (pVITRO-IP-10) was constructed, and biodegradable cationic heparin-polyethyleneimine (HPEI) nanogels were prepared to deliver pVITRO-IP-10 into SKOV3 human ovarian cancer cells. Transfection efficiency was detected by expression profiling of green fluorescent protein. The expression of IP-10 was determined using RT-PCR and western blot analysis. In vitro, cell proliferation was evaluated by MTT assay. Apoptosis was examined by Hoechst33258/PI staining and flow cytometry assays. The effect on the inhibition of angiogenesis was evaluated by tube formation assay using human umbilical vein endothelial cells (HUVECs). Moreover, a SKOV3 intraperitoneal ovarian carcinomatosis model was established to investigate the antitumor activity of HPEI+pVITRO-IP-10 complexes in nude mice. Tumor weights were evaluated during the treatment course. Cell proliferation and apoptosis were evaluated by Ki-67 immunochemical staining and TUNEL assay, and the antiangiogenic effect of pVITRO-IP-10 was assessed by CD31 immunochemical staining and alginate-encapsulated tumor cell assay. pVITRO-IP-10 was efficiently transfected into SKOV3 cells by HPEI nanogels. Intraperitoneal admin-
\end{abstract}

Correspondence to: Dr Xia Zhao, Department of Gynecology and Obstetrics, Key Laboratory of Obstetric and Gynecologic and Pediatric Diseases and Birth Defects of Ministry of Education, West China Second Hospital; State Key Laboratory of Biotherapy and Cancer Center, Sichuan University, Chengdu 610041, Sichuan, P.R. China E-mail:xia-zhao@126.com

Key words: angiogenesis, apoptosis, gene therapy, IP-10, HPEI, ovarian cancer istration of HPEI+pVITRO-IP-10 complexes led to effective growth inhibition of ovarian cancer, in which tumor weight decreased by $\sim 69.92 \%$ in the treatment group compared with that in the empty vector control group. Meanwhile, decreased cell proliferation, increased tumor cell apoptosis and reduction in angiogenesis were observed in the HPEI+pVITRO-IP-10 group compared with those in the control groups. These results indicated that HPEI nanogel delivery of pVITRO-IP-10 may be of value in the treatment against human ovarian cancer.

\section{Introduction}

Ovarian cancer is one of the most common and lethal gynecologic neoplasm, and presently ranks fifth in causing female cancerrelated mortality (1). The overall 5-year relative survival rate is no more than $50 \%$ (2). Due to its few and imperceptible early symptoms, ovarian cancer is mostly at advanced stage during the initial diagnosis. Although patients may initially respond to the combination treatment of surgical and chemical therapies, and various new chemotherapeutics have been developed for the treatment of ovarian cancer, most of the patients will inevitably die from relapse and the development of chemotherapy-resistant recurrence (3). Drug resistance and severe side effects remain unavoidable obstacles for chemotherapeutic approaches. Development of new approaches to overcome the therapeutic dilemma is of great urgency.

Interferon $\gamma$-inducible protein of $10 \mathrm{kDa}$ (IP-10/CXCL10), a member of the non-ELR-CXC chemokine superfamily, is known to a highly inducible chemoattractant binding to the CXCR3 chemokine receptor $(4,5)$. According to present studies, IP-10 has been demonstrated to be a pleiotropic molecule eliciting potent biological effects, including stimulation of monocytes, natural killer (NK) cells and T-cell, regulation of T-cell and bone marrow progenitor maturation, modulation of adhesion molecule expression as well as inhibition of angiogenesis (6). It has also been reported that IP-10 may inhibit human non-small cell lung cancer (NSCLC) tumorigenesis and spontaneous metastases (7). Currently, much interest is focused on the anti-neoplastic effects of IP-10 (8). IP-10 has been found to possess anti-tumor and anti-metastatic properties by immunological, antiangiogenic and anti-neoplastic mechanisms $(9,10)$. 
In the present study, we constructed this recombinant plasmid expressing IP-10 (pVITRO-IP-10) to test its antitumor effect on ovarian cancer.

In addition, the delivery system is a crucial factor affecting the effectiveness of therapy for ovarian cancer. Polyethyleneimine (PEI) has emerged as one of the most effective nonviral gene carrier agent. Currently, commercial PEI25K (25 kg/mol) is widely considered to be the gold standard for assessing the transfection efficiency of new polymer-based gene carriers $(11,12)$. However, PEI has the shortcoming of inducing marked aggregation of erythrocytes and hemolysis $(13,14)$. Moreover, PEI whose transfection efficiency comes with relatively high cytotoxicity is not biodegradable. In our previous study, PEI2K was chemically conjugated with heparin to form a novel biodegradable cationic nanogel, named HPEI. The novel biodegradable cationic HPEI nanogels were then used to effectively deliver a plasmid expressing vesicular stomatitis virus matrix protein (pVSVMP) into C-26 cells in vitro and in vivo, where the complex efficiently suppressed the growth of abdominal and pulmonary metastases in C-26 colon carcinomas in BALB/c mice (15). HPEI nanogels have also been used to transfect the novel FILIP1LDC103-p gene into SKOV3 cells efficiently (16). In the present study, biodegradable cationic HPEI nanogels were used as gene carriers to deliver a recombinant plasmid encoding IP-10 to treat a SKOV3 intraperitoneal ovarian carcinomatosis in nude mice, potential enhanced effect of IP-10 on ovarian cancer was expected.

\section{Materials and methods}

Cell culture. Human epithelial serous cystadenocarcinoma SKOV3 cells were obtained from American Type Culture Collection (Rockville, MD, USA) and cultured in DMEM (Gibco, Carlsbad, CA) culture medium supplemented with $10 \%$ FCS, $2 \mathrm{mM}$ L-glutamine, $100 \mathrm{U} / \mathrm{ml}$ penicillin, and $100 \mu \mathrm{g} / \mathrm{ml}$ streptomycin. Cells were incubated in a humidified atmosphere containing $5 \% \mathrm{CO}_{2}$ at $37^{\circ} \mathrm{C}$ and passaged every 4 days at a split ratio of $1: 3$.

HUVECs were isolated from human umbilical vein vascular wall as described previously (17). Briefly, the human umbilical vein vascular wall was digested with collagenase IV at $37^{\circ} \mathrm{C}$ for $10 \mathrm{~min}$, and the homogenate was centrifuged at $750 \mathrm{~g}$ for $10 \mathrm{~min}$. Cells were suspended and seeded on fibronectin-coated plates and cultured in Earle's salts medium supplemented with $10 \%$ fetal calf serum (FCS).

Preparation of plasmids. A dual promoter plasmid, pVITRO2neo-mcs (Invitrogen, San Diego, CA, USA) was utilized to express IP-10 genes, which is a common expression vector in gene therapy (18). The recombinant plasmid was named pVITRO-IP-10. The pVITRO2-neo-mcs plasmid cDNA without IP-10 was used as the empty-vector control (hereafter called E-p). The plasmids were purified using Qiagen Endo-free Giga kit (Qiagen, Hilden, Germany) following the manufacturer's instructions (19).

HPEI preparation and plasmid transfection. Biodegradable HPEI nanogels were synthesized as previously described (15). In brief, $0.05 \mathrm{~g}$ of heparin was first dissolved in 2-(N-morpholino) ethanesulfonic acid (MES) buffering agent (100 ml, $50 \mathrm{mM})$;
$0.02 \mathrm{~g}$ of 1-ethyl-3-(3-dimethylaminopropyl) carbodiimide (EDC) and $0.03 \mathrm{~g}$ of N-hydroxysuccinimide (NHS) were subsequently added to the solution to activate heparin's carboxylic acid groups. After $20 \mathrm{ml}$ of PEI2K solution $(7.5 \mathrm{mg} / \mathrm{ml})$ was dropped into the solution, the reaction was carried out at room temperature overnight. The resulting HPEI nanogels were then dialyzed in double-distilled water for 3 days. Later, HPEI nanogels were filtered using a syringe filter, then adjusted to a final concentration of $1.0 \mathrm{mg} / \mathrm{ml}$ and stored at $4^{\circ} \mathrm{C}$.

SKOV3 ovarian cancer cells $\left(2.0 \times 10^{5}\right)$ were grown on 6-well plates in DMEM medium and cultured for $24 \mathrm{~h}$. Twenty micrograms of HPEI and $2 \mu \mathrm{g}$ of pVITRO-IP-10 was diluted in $1 \mathrm{ml}$ of DMEM medium without antibiotic or serum, and then combined at a ratio of 10:1. The combinations were transfected to cells in triplicate and incubated overnight to $80 \%$ confluence. Meanwhile, the medium alone and HPEI nanogels alone were used as control agents. After 4-6 h, the medium was replaced by $2 \mathrm{ml}$ of DMEM medium with $10 \%$ FCS, 2 mM L-glutamine, $100 \mathrm{U} / \mathrm{ml}$ penicillin, and $100 \mu \mathrm{g} / \mathrm{ml}$ streptomycin. After $48 \mathrm{~h}$ of incubation, the cells and supernatants were collected for further assay.

Detection of transfection efficiency. In order to evaluate the transfection efficiency of HPEI in vitro, $2 \mu \mathrm{g}$ of green fluorescent protein (GFP) plasmid was encapsulated with HPEI to transfect SKOV3 cells. The GFP expression profile was detected as described in our previous studies (20). In brief, SKOV3 cells $\left(1 \times 10^{5}\right.$ cells/well) were seeded on 6 -well plates. Before transfection, the DMEM medium in each well was replaced with $0.8 \mathrm{ml}$ of fresh serum-free medium, and then the HPEI:GFP complex was added and left to mix in serum-free medium at $37^{\circ} \mathrm{C}$ overnight. Then the complete DMEM medium was added, and the plate was maintained at $37^{\circ} \mathrm{C}$ for $48 \mathrm{~h}$, after which we observed green fluorescence expression with a fluorescence inverted microscope (Nikon Eclipse 80i, Japan). The transfection efficiency was determined based on the percentage of cells expressing GFP. The number of GFP-expressing cells divided by the total cell quantity under the microscope was defined as the transfection efficiency. Cell counting was performed randomly in the microscopic observation scope under magnifications $\mathrm{x} 10$ with 3 repeats. All data are presented as the mean \pm standard deviation (SD).

$R T-P C R$. The IP-10 primers were designed based on their cDNA sequences, upstream IP-10 primer: 5'-ATGAATCAAACTGCC ATTCTG-3'; and downstream IP-10 primer: 5'-TTAAGGAGA TCTTTTAGACCTTTCC-3'.

Total RNA was purified using the TRIzol reagent (Invitrogen, Carlsbad, CA, USA), according to the manufacturer's instructions. RT-PCR was performed as follows: incubation at $50^{\circ} \mathrm{C}$ for $40 \mathrm{~min}$ and denaturation at $94^{\circ} \mathrm{C}$ for $2 \mathrm{~min}$, followed by a standard PCR regime of $94^{\circ} \mathrm{C}$ for $30 \mathrm{sec} 53^{\circ} \mathrm{C}$ (IP-10) for $30 \mathrm{sec}$, and $72^{\circ} \mathrm{C}$ for $40 \mathrm{sec}$ (30 cycles). Each RT-PCR product $(5 \mu \mathrm{l})$ was analyzed by agarose gel (1.5\%) electrophoresis. The housekeeping gene GAPDH served as the internal control. The density of PCR bands was quantified by Quantity One software.

Western blot analysis. Western blot analysis was employed to determine the expression level of IP-10 in vitro and in vivo. Cells or tumor tissue were lysed in RIPA lysis buffer containing 
$50 \mathrm{mM}$ Tris- $\mathrm{HCl}$ (pH 7.4), $0.25 \%$ sodium deoxycholate, $150 \mathrm{mM}$ $\mathrm{NaCl}, 0.1 \%$ SDS, $1 \%$ NP-40, $1 \mathrm{mM}$ EDTA, $1 \mathrm{mM} \mathrm{NaF}$, and $1 \mathrm{mM}$ $\mathrm{Na}_{3} \mathrm{VO}_{4}$, supplemented with proteinase inhibitor $(1 \mathrm{mM}$ cocktail plus $1 \mathrm{mM}$ PMSF, Sigma, St. Louis, MO). The lysed product was then centrifuged at $15,000 \mathrm{rpm}, 4^{\circ} \mathrm{C}$ for $30 \mathrm{~min}$. Protein concentrations were measured with Bio-Rad protein assay kits (Bio-Rad Laboratories, Hercules, CA). Equal amounts of protein $(40 \mu \mathrm{g})$ were loaded onto a $12 \%$ SDS-PAGE gel for electrophoresis and transferred onto polyvinylidene difluoride (PVDF) membrane (Millipore, Bedford, MA). After being blocked in 5\% skim milk for $1 \mathrm{~h}$, the membranes were incubated with anti-IP-10 antibody (1:100 dilution, Abcam 9807) at $4^{\circ} \mathrm{C}$ overnight. The membranes were then incubated with goat anti-rabbit secondary antibody at 1:5,000 dilution (Abcam, Cambridge, MA) in PBS with Tween- 20 for $1 \mathrm{~h}$. Targeted protein bands were detected by the enhanced chemiluminescence detection system (Pierce Biotech Inc., Rockford, IL) according to the manufacturer's instructions. GAPDH served as the protein loading control. Bands were analyzed using Quantity One 4.52 software (Bio-Rad).

Assessment of proliferation in vitro. The effect of pVITROIP-10 on cell proliferation was measured by MTT assay (21). SKOV3 transfection procedure was as mentioned above, then $10 \mathrm{ml}$ of $5 \mathrm{mg} / \mathrm{ml}$ MTT (pH 4.7) was added to each well, and the cells were incubated for another $4 \mathrm{~h}$. The supernatant was then removed; $100 \mathrm{ml} \mathrm{DMSO/well} \mathrm{was} \mathrm{added} \mathrm{followed} \mathrm{by}$ shaking for $15 \mathrm{~min}$. Absorbance at $490 \mathrm{~nm}$ was measured with a micro-plate reader. Independent experiments were performed in triplicate. Inhibition of cell proliferation (\%) was assessed using the formula: cell inhibition $(\%)=(1-\mathrm{A} 490$ of treated cells/A490 of control cells) x $100 \%$.

Assessment of apoptosis in vitro. Cell apoptosis was analyzed by Hoechst 33258 staining. Briefly, $48 \mathrm{~h}$ after transfection, cells were immersed in $0.5 \mathrm{ml}$ of methanol for $15 \mathrm{~min}$, followed by two PBS rinses. Then the cells were stained with $1 \mu \mathrm{g} / \mathrm{ml}$ Hoechst 33258 compounds in a dark chamber at room temperature for $10 \mathrm{~min}$ and again rinsed two times with PBS, then stained with 1X PI $500 \mu \mathrm{l}$ for $10 \mathrm{~min}$ and rinsed two times with PBS again. Cells were analyzed by fluorescence microscopy using excitation and emission wavelengths of 350 and $460 \mathrm{~nm}$, respectively (22).

Quantitative evaluation of cellular apoptosis was performed by flow cytometric analysis. Briefly, after processing in 6-well plates as described above, the floated cells were discarded while the attached cells were trypsinized and thereafter washed twice with cold PBS. Cells were resuspended in prediluted binding buffer and stained with propidium iodide $(10 \mu \mathrm{g} / \mathrm{ml}$, BD Pharmingen, San Jose, CA, USA) for $10 \mathrm{~min}$ in the dark at room temperature, and the mixtures were immediately analyzed by flow cytometry (23).

Assessment of antiangiogenesis in vitro. Antiangiogenesis effect of pVITRO-IP-10 was evaluated by tube formation of human umbilical vein endothelial cells (HUVEC) $(24,25)$. HUVEC were cultured as described above. Tube formation assay is reported as an effectively model for assessment of antiangiogenesis in vitro. Briefly, Matrigel was incubate on ice at $4^{\circ} \mathrm{C}$ overnight. Pre-cooled matrigel $(150 \mu \mathrm{l})$ was transfered to a 48 -well plate on ice and allowed to solidify at $37^{\circ} \mathrm{C}$ for
$1 \mathrm{~h}$. HUVEC were counted and diluted to $4 \times 10^{5}$ cells $/ \mathrm{ml}$ in cell culture media. The molecules were diluted to required concentrations in cell culture media. The diluted HUVEC were transfer to wells of a 96-well plate. Respective diluted molecules were added to protein solution by pipetting them up and down three times, then ensuring the final concentration as 1x mix. The mixture (200 $\mu \mathrm{l})$ was transfered to a 48 -well plate and incubated at $37^{\circ} \mathrm{C}, 5 \% \mathrm{CO}_{2}$ for 3 to $30 \mathrm{~h}$. Endothelial tube size and numbers were recorded under a microscope and photograph. The increase or decrease in the formation of tubes in the test wells as compared to control wells indicated whether the molecule was angiogenic or anti-angiogenic.

Tumor xenograft model and animal treatment. All animal research procedures were approved by the Institutional Animal Care and Use Committee of Sichuan University (Chengdu, China). Female athymic BALB/c nude mice, 6-8 weeks old, were used to establish an intraperitoneal carcinomatosis model using a previously-established method with some modifications (26). In brief, $5 \times 10^{6}$ SKOV3 cells in $100 \mu 1$ DMEM medium (free of serum and antibiotics) were injected subcutaneously into the dorsal sides of 6 mice. When the diameter of the resulting subcutaneous tumors reached $\sim 10 \mathrm{~mm}$, the tumor nodes were collected and minced into tiny particles $\left(<1 \mathrm{~mm}^{3}\right)$ by sterile stainless screen cloth. The tumor particles were then resuspended by serum-free medium to a final volume of $15 \mathrm{ml}$, and 30 mice were inoculated intraperitoneally with $0.5 \mathrm{ml}$ of the mixture. Mice were assigned randomly to one of the following groups (five per group): a) untreated, $100 \mathrm{ml}$ of $5 \%$ glucose solution; b) HPEI, $50 \mathrm{ml}$ of HPEI in $50 \mathrm{ml}$ of $5 \%$ glucose solution; c) HPEI \pm E-p, $5 \mu \mathrm{g}$ of pVITRO2-neo-mcs of HPEI in $50 \mathrm{ml}$ of $5 \%$ glucose solution; and (d) HPEI+pVITRO-IP-10, $5 \mu \mathrm{g}$ of pVITRO-IP-10 and $50 \mathrm{ml}$ of HPEI in $50 \mathrm{ml}$ of $5 \%$ glucose solution; intraperitoneal administration was initiated 5 days after inoculation. The HPEI/DNA complexes were prepared at room temperature before administration. The mice received therapy every 3 days and were sacrificed after 12 treatments. Intraperitoneal tumors were resected and weighed immediately to assess antitumoral efficacy.

Histological analysis. Each dissected tumor node group was divided into two parts. One was fixed in $10 \%$ formalin for $48 \mathrm{~h}$ and then embedded in paraffin. The other was stored at $-80^{\circ} \mathrm{C}$ for frozen section and protein extraction. Sections $(3-5 \mu \mathrm{m})$ were made of the paraffin-embedded tumors of each group. Then the paraffin sections were stained with hematoxylin and eosin (HE) and examined by an experienced pathologist using light microscopy to confirm histology. Immunohistochemistry staining of IP-10, and Ki67 was performed as described previously (27). Briefly, deparaffinized tumor sections were immersed in $10 \mathrm{mM}$ citrate buffer (pH 6.0) and then heated in an autoclave for $5 \mathrm{~min}$ in saturated steam for antigen retrieval. Endogenous peroxidase activity was quenched in $3 \% \mathrm{H}_{2} \mathrm{O}_{2}$ for $10 \mathrm{~min}$, and the non-specific binding sites of reagents were subsequently blocked with homeotypic nonimmunoglobulin of the secondary antibody at $37^{\circ} \mathrm{C}$ for $20 \mathrm{~min}$. Then tumor sections were incubated with the primary antibody (R\&D Systems, Minneapolis, $\mathrm{MN})$ at $4^{\circ} \mathrm{C}$ overnight. Tumor sections were incubated with the biotinylated secondary antibody at $37^{\circ} \mathrm{C}$ for $40 \mathrm{~min}$, followed by sequential incubation with a streptavidin-biotin-horseradish 
peroxidase complex for $40 \mathrm{~min}$ at $37^{\circ} \mathrm{C}$. Colorimetric detection was performed with diaminobenzidine.

TUNEL assay for apoptotic cells. TUNEL staining was performed to analyze apoptotic cells in tumor tissues using apoptotic cell kits according to the manufacturer's protocol (Promega, Madison, WI) (16). The apoptosis index was calculated as the average percentage of green fluorescence-positive cells in 10 random fields from different sections at magnification $\mathrm{x} 400$.

Alginate-encapsulated tumor cell assay. To explore inhibition of angiogenesis, we performed an alginate-encapsulation assay. Briefly, SKOV3 cells were resuspended in a $1.5 \%$ solution of alginate (Sigma) and added dropwise into a solution of $250 \mathrm{mM} \mathrm{CaCl}_{2}$; an alginate bead was formed containing $1 \times 10^{5}$ cells. Two beads were then implanted subcutaneously in the backs of every nude mouse. Eighteen mice were then grouped and treated as described earlier. Treatment was initiated on the same day the beads were implanted. After two weeks, mice were injected intravenously with $0.2 \mathrm{ml}$ of a $50 \mathrm{mg} / \mathrm{kg}$ fluorescein isothiocyanate (FITC)-dextran (Sigma) solution. Alginate beads were photographed after being exposed surgically and then rapidly removed $20 \mathrm{~min}$ after FITC-dextran injection. The uptake of FITC-dextran was measured as described by Hoffmann et al (28).

Microvessel density assay. The frozen tumor masses stored in liquid nitrogen were sliced into $5-\mu \mathrm{m}$ sections for quantification of microvessel density (MVD) with CD31 staining by the immunohistochemical ABC method, following the protocol of the manufacturer with a few modifications. Briefly, after fixing frozen sections in cold acetone for $20 \mathrm{~min}$, rabbit serum was used in the blocking steps. Subsequently, endogenous peroxidase was blocked with $0.3 \% \mathrm{H}_{2} \mathrm{O}_{2}$. Sections were then incubated in monoclonal CD31 primary antibody of rat anti-mouse PECAM-1 diluted 1:400 overnight at $4^{\circ} \mathrm{C}$. After incubating and washing in PBS, sections were incubated in biotinylated rabbit anti-rat IgG (secondary antibody, 1:100 dilution) at $37^{\circ} \mathrm{C}$ for $40 \mathrm{~min}$. The sections were then treated with streptavidin-biotin reagents, resulting in the brown staining of microvessel endothelial cells. Then MVD was determined by examining vascular hot spots as described previously (29). Three vascular tumor areas without necrosis were selected at high magnification (x200), and the three values were averaged for each sample.

Evaluation of possible adverse effects. For mice treated with pVITRO-IP-10 DNA complex, gross measures such as weight loss, diarrhea, toxic death, life span, behavior, and feeding were investigated in particular for potential signs of toxicity. Heart, liver, spleen, lung, and kidney tissues were fixed in $10 \%$ buffered formalin solution and embedded in paraffin. Sections of 3-5 $\mu \mathrm{m}$ were stained with hematoxylin and eosin $(\mathrm{H} \& \mathrm{E})$ and observed by two different experienced pathologists in a blinded manner.

Statistical analysis. All experimental data are expressed as mean \pm SD. One-way ANOVA, followed by Tukey's test for multiple comparisons, was performed with SPSS software (version 15.0 for Windows). Dunnett's t-test (2-sided) was employed as needed. Statistical significance was determined by the log-rank test. Significance was defined as $\mathrm{p}<0.05$.

\section{Results}

Transfection efficiency and expression of IP-10 in vitro and in vivo. The transfection efficiency of HPEI in vitro was tested by green fluorescent protein (GFP). The transfection efficiency, based on the percentage of cells expressing GFP, is $\sim 48.8 \% \pm 5.15$, shown in Fig. 1A.

The in vitro expression of the IP-10 gene in transfected SKOV3 cells was confirmed using RT-PCR and western blot analysis. As shown in Fig. 1B, the expression of IP-10 in SKOV3 cells transfected with HPEI+pVITRO-IP-10 could be detected, whereas there was no expression of IP-10 in SKOV3 cells from the untreated, HPEI, or HPEI+E-p groups

To examine the expression of IP-10 in vivo, an intraperitoneal ovarian carcinoma model was established in nude mice, which were then treated with HPEI+pVITRO-IP-10, HPEI+E-p, HPEI, or 5\% glucose. Tumors were collected for RT-PCR and western blotting on the third day after the final treatment. The level of IP-10 mRNA and expression of IP-10 protein were detected in tumor tissues from the HPEI+pVITRO-IP-10 group, whereas there was no expression in the HPEI, E-p, and 5\% glucose groups (Fig. 1C). In addition, immunochemical staining of IP-10 in tumor tissues from the HPEI+pVITRO-IP-10 group also suggested positive expression, as shown in Fig. 1D.

Inhibition of proliferation and increased apoptosis induced by pVITRO-IP-10 in vitro. The effect of pVITRO-IP-10 on cell proliferation was measured by MTT assay. The inhibition rate of SKOV3 cell proliferation $72 \mathrm{~h}$ after transfection with HPEI reached $45.3 \%$ in pVITRO-IP-10 treated samples, while it is $21.7 \%$ in E-p treated samples, and $9.6 \%$ in samples treated only with HPEI, in comparison with untreated samples. Cell viability was significantly lower in the groups treated with pVITRO-IP-10 than in the other three control groups (untreated, HPEI alone, and E-p), as shown in Fig. 2A ( $\mathrm{p}<0.05)$.

Hoechst 33258/PI staining was used to test the apoptosis. The pVITRO-IP-10 treated cells resulted in more morphological changes compared with controls. As shown in Fig. 2B, bright blue-fluorescent condensed nuclei (intact or fragmented), decreased cell volume, blebbing, condensation of nuclear chromatin, nuclear fragmentation, and apoptotic bodies were common in the pVITRO-IP-10 treated cells. Those cells also displayed prominent red fluorescence. However, these changes were less prominent, and slight bright blue or red fluorescence appeared in the untreated, HPEI alone, and E-p groups.

In addition, the number of apoptotic and non-apoptotic cells were quantitated by flow cytometric analysis. The results correlated strongly with those in the staining patterns observed with fluorescence microscopy. The percentage of apoptotic cells was significantly higher in the pVITRO-IP-10 samples (56.4\%) compared with samples treated with E-p $(22.3 \%)$, HPEI (11.8\%), or untreated (5.1\%) as shown in Fig. 2C.

Inhibition of angiogenesis in vitro. We tested the ability of IP-10 to inhibit endothelial cell activity in vitro using tube formation assay. Untreated HUVECs display a healthy tubular network formation. In the case of HUVECs treated by HPEI alone or 


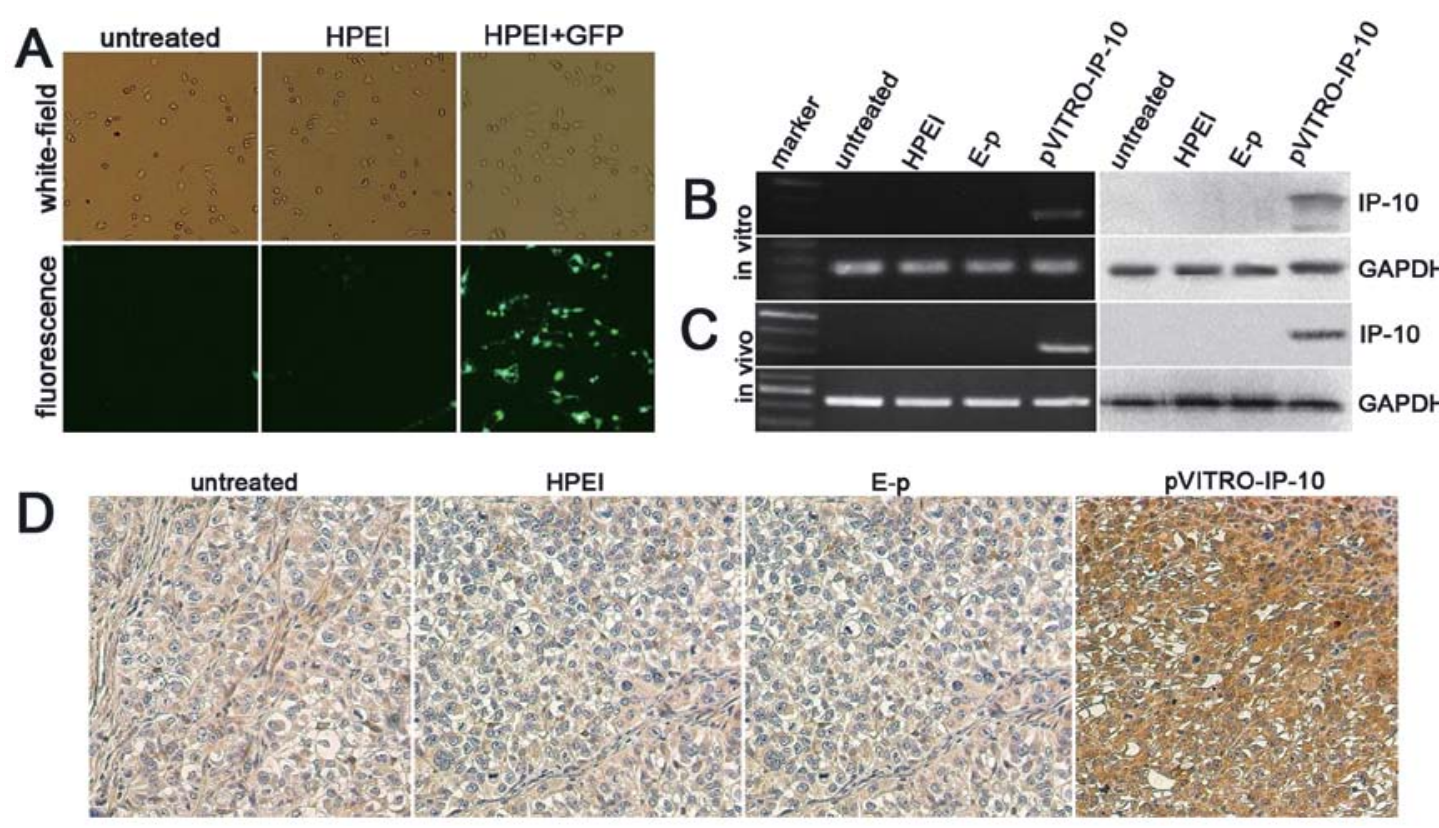

Figure 1. Expression of IP-10 in vitro and in vivo. (A) Identification of the transfection efficiency is based on the percentage of SKOV3 cells expressing GFP in vitro; at $\sim 48.8 \pm 5.15 \%$. (B) In vitro, the expression of IP-10 in SKOV3 cells transfected with HPEI+pVITRO-IP-10 could be detected by RT-PCR and western blot analysis, whereas there was no expression in untreated, HPEI, or E-p groups. The housekeeping gene GAPDH served as internal control. (C) In vivo an intraperitoneal ovarian carcinoma model was established in nude mice, the level of IP-10 mRNA and expression of IP-10 (10-kDa) protein were detected in tumor tissues from the HPEI+pVITRO-IP-10 group, whereas there was no expression in the HPEI, E-p, and untreated groups. 37-kDa GAPDH served as internal control. (D) Immunochemical staining of IP-10 in tumor tissue.

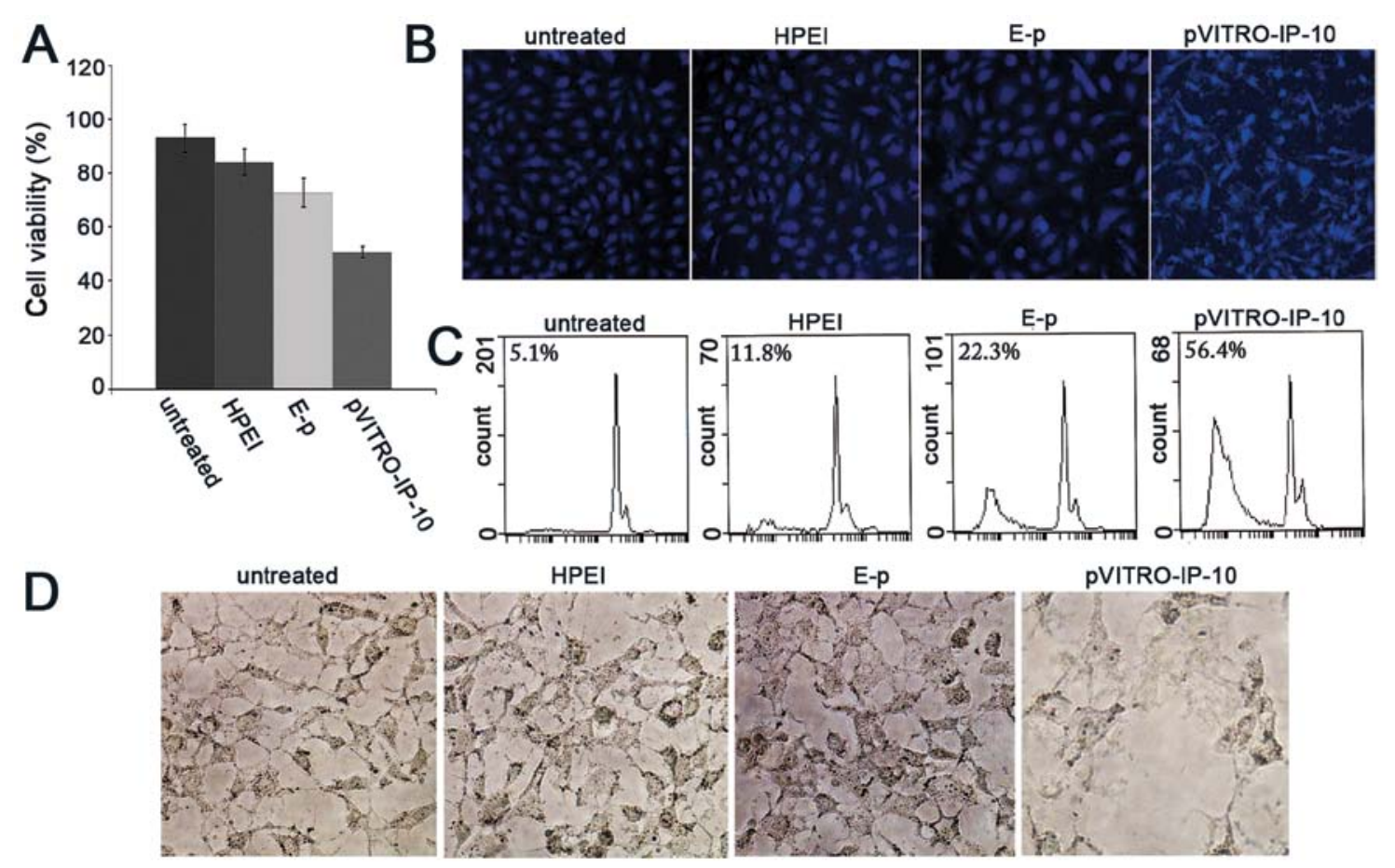

Figure 2. Inhibition of proliferation, apoptosis and antiangiogenesis induced by pVITRO-IP-10 in vitro. (A) SKOV3 cell proliferation was determined by MTT assay. Seventy-two hours after transfection cell viability was significantly lower in the groups treated with HPEI+pVITRO-IP-10 than in the other three control groups (untreated, HPEI alone, and E-p). (B) Bright blue-fluorescent condensed nuclei (intact or fragmented), decreased cell volume, blebbing, condensation of nuclear chromatin, nuclear fragmentation, and apoptotic bodies were common in the pVITRO-IP-10 treated cells, and less prominent in the untreated, HPEI or E-p groups. (C) The percentage of apoptotic cells was confirmed by flow cytometric analysis as significantly higher in the pVITRO-IP-10 samples (56.4\%) compared with samples treated with E-p (22.3\%), HPEI (11.8\%), or untreated (5.1\%). (D) Obvious inhibition of HUVECs tubular network formation was found in pVITRO-IP-10 treated group, while they display healthy tubular network formation in untreated, HPEI or HPEI+E-p groups.

HPEI+E-p supernatant, there was tubular network formation almost similar to that of the control. In the case of HUVECs treated with pVITRO-IP-10 supernatant, we found obvious inhibition of tubular network formation (Fig. 2D). 

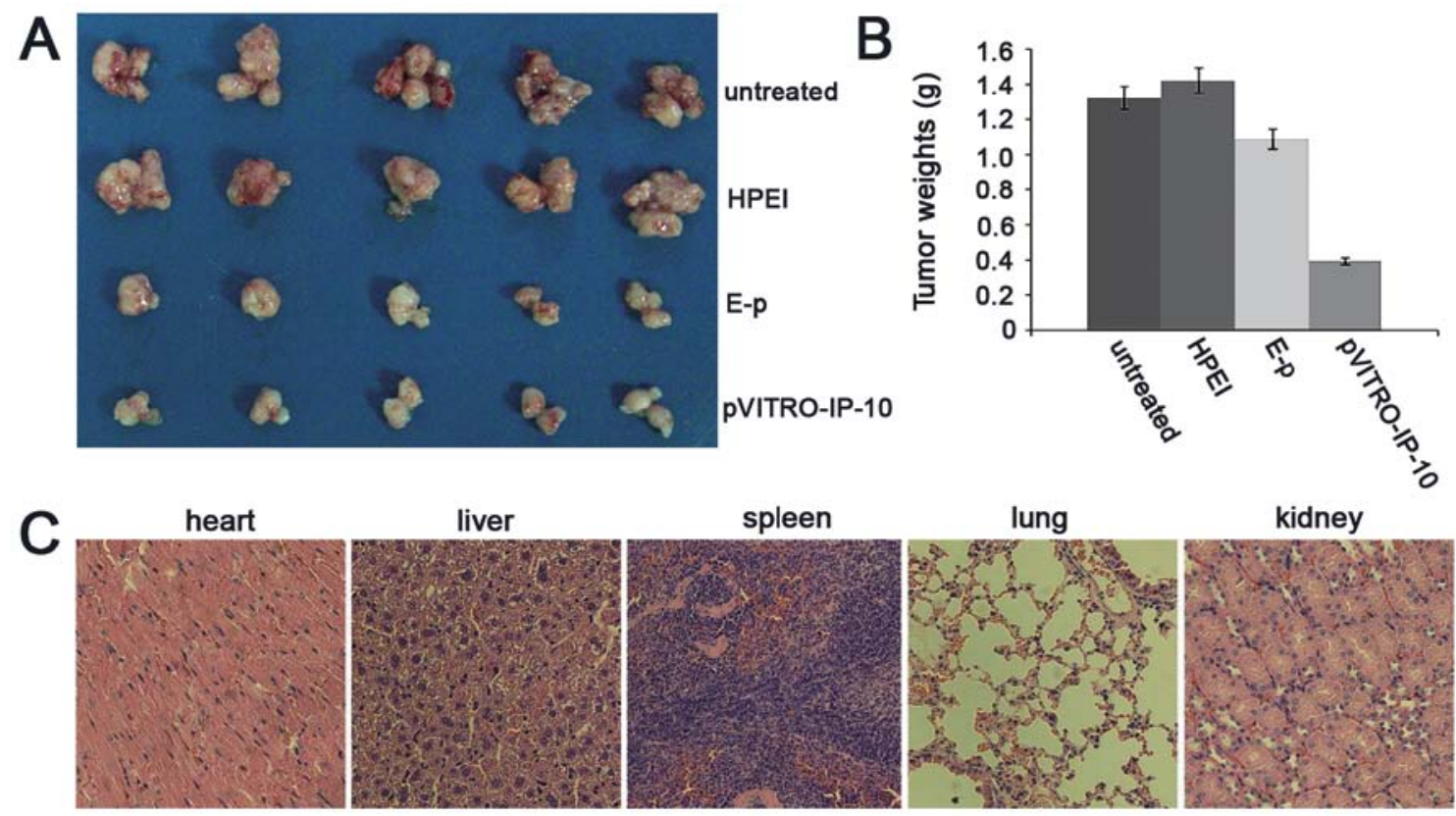

Figure 3. Inhibition of intraperitoneal ovarian cancer xenograft growth in nude mice by pVITRO-IP-10 and observation of toxicity in vivo. (A) Tumor from HPEI+pVITRO-IP-10 treated mice showed significant difference compared with tumors from the other control groups ( $<<0.01$ ). (B) All intraperitoneal tumor nodules were collected carefully; each group with 5 mice. The average tumor weights were $1.33 \pm 0.41$ (untreated), $1.43 \pm 0.29$ (HPEI), $1.10 \pm 0.20$ (HPEI+E-p), and $0.40 \pm 0.04 \mathrm{~g}$ (HPEI+pVITRO-IP-10). (C) Histologically, no apparent pathological differences were noted between the treated and control groups in the HE-stained main organs, including heart, liver, spleen, lung, and kidney.
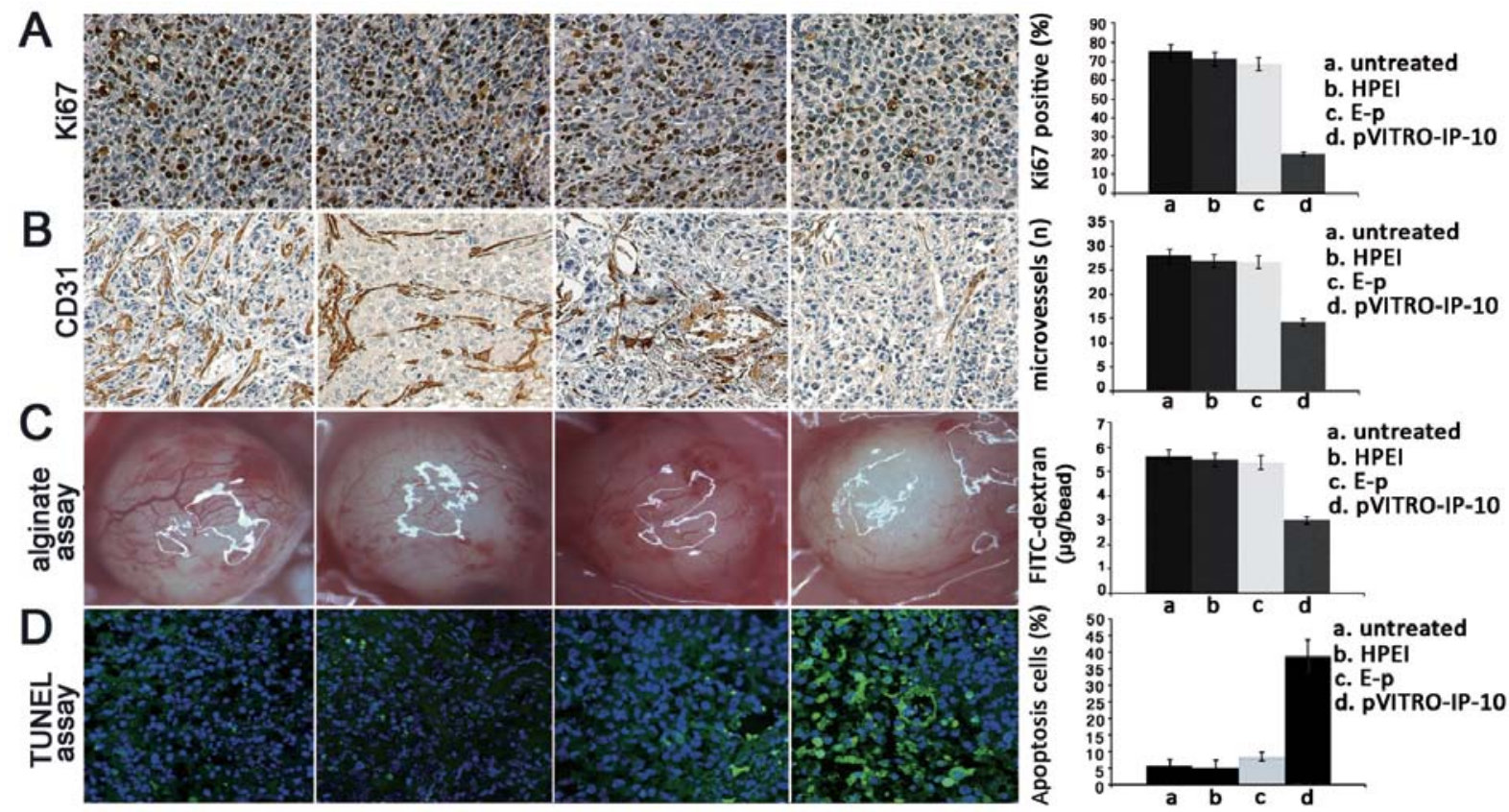

Figure 4. Inhibition of cell proliferation, suppression of angiogenesis and induction of cell apoptosis by pVITRO-IP-10 in vivo. (A) Ki-67 immunochemical staining was performed to detect the cell proliferation inhibition. The Ki67-positive cells were 75.3 \pm 6.67 (untreated) vs. $71.34 \pm 6.49$ (HPEI) vs. $68.5 \pm 4.50$ (HPEI + E-p) vs. 20.94 \pm 3.51 (HPEI+pVITRO-IP-10) vs. respectively. (B) The antiangiogenic effect of HPEI+pVITRO-IP-10 was detected by CD31 immunochemical staining. This staining revealed a significant reduction of MVD in tumor tissues from the HPEI+pVITRO-IP-10 treated groups compared with the untreated, HPEI, and HPEI + E-p groups. The MVD were 27.91 \pm 1.67 (untreated) vs. $26.97 \pm 1.23$ (HPEI) vs. 26.74 \pm 0.85 (HPEI + E-p) vs. 14.32 \pm 1.36 (HPEI+pVITRO-IP-10) vs. respectively. (C) Antiangiogenesis activity was also detected by the alginate-encapsulated tumor cell assay. There were fewer newborn blood vessels in the alginate bead nude mice treated with HPEI+pVITRO-IP-10 than in those from the control groups. The FITC-dextran uptake were $5.62 \pm 0.46 \mu \mathrm{g} / \mathrm{bead}$ (untreated) vs. $5.49 \pm 0.45 \mu \mathrm{g} / \mathrm{bead}(\mathrm{HPEI})$ vs. $5.37 \pm 0.37 \mu \mathrm{g} / \mathrm{bead}$ (HPEI+E-p) vs. $2.99 \pm 0.16 \mu \mathrm{g} / \mathrm{bead}$ (HPEI+pVITRO-IP-10), respectively. Compared with the three control groups, the levels of FITC-dextran uptake were significantly lower in mice treated with HPEI+pVITRO-IP-10. (D) Apoptosis was detected by TUNEL assay. In tumor tissues, apoptotic cells were stained with bright green fluorescence, while normal live cells were stained by DAPI in bland blue. The TUNEL assay showed many apoptotic cells in the tumor sections of the HPEI+pVITRO-IP-10 groups, however, there were few such cells in the three control groups. The apoptotic index, the ratio of bright green-fluorescence cells to total cells, revealed that tumors in the HPEI+pVITRO-IP-10 groups had significantly more TUNEL-positive nuclei than tumors in the untreated, HPEI, or HPEI + E-p groups, apoptosis ratio was $5.79 \pm 1.67$ (untreated) vs. $5.11 \pm 1.69$ (HPEI) vs. $8.38 \pm 2.16$ (HPEI+E-p) vs. 39.92 \pm 4.84 (HPEI+pVITRO-IP-10), which revealed that tumors in the HPEI+pVITRO-IP-10 groups had significantly more TUNEL-positive nuclei than tumors in the untreated, HPEI, or HPEI + E-p groups. 
Inhibition of intraperitoneal ovarian cancer xenograft growth in nude mice. To evaluate the effect of HPEI+pVITRO-IP-10 on suppressing ovarian cancer growth in vivo, we established an intraperitoneal xenograft model of human ovarian cancer. All intraperitoneal tumor nodules were collected carefully; each group with 5 mice. As shown in Fig. 3A, the average tumor weights were $1.33 \pm 0.41$ (untreated), $1.43 \pm 0.29$ (HPEI), $1.10 \pm 0.20$ (HPEI+E-p), and $0.40 \pm 0.04 \mathrm{~g}$ (HPEI+pVITRO-IP-10), $(\mathrm{p}<0.01)$. It suggested that HPEI+pVITRO-IP-10 reduced tumor weight by $69.92 \%$ compared with the controls (Fig. 3B).

Observation of toxicity. To determine potential side effects during treatment, we looked for signs of possible toxicity. No gross abnormalities were found regarding weight loss, feeding, or behavior in the treated groups. Furthermore, histologically, no apparent pathological differences were noted between the treated and control groups in the HE-stained organs, including heart, liver, spleen, lung, and kidney (Fig. 3C).

Inhibition of cell proliferation in vivo. To explore potential mechanisms underlying the antitumor effect of HPEI+ pVITRO-IP-10 in vivo, we performed Ki-67 immunohistochemistry to investigate whether the antitumor effect of HPEI+pVITRO-IP-10 correlated with cell proliferation inhibition. The reduction of $\mathrm{Ki}-67$ expression relative to the three control groups was obvious in the tumors of the HPEI+pVITRO-IP-10 treated groups. The Ki67-positive cells were $75.3 \pm 6.67$ (untreated) vs. $71.34 \pm 6.49$ (HPEI) vs. $68.5 \pm 4.50$ (HPEI+E-p) vs. 20.94+3.51 (HPEI+pVITRO-IP-10) $(\mathrm{p}<0.05$; Fig. 4A).

Suppression of angiogenesis in vivo. Frozen sections were stained by CD31 antibody to investigate the antiangiogenic effect of HPEI+pVITRO-IP-10. Angiogenesis in tumor tissues was evaluated by microvessel density (MVD) in different sections stained with an antibody reactive to CD31, which has high specific affinity for vascular endothelial cells. This staining revealed a significant reduction of MVD in tumor tissues from the HPEI+pVITRO-IP-10 treated groups compared with the untreated, HPEI, and HPEI+E-p groups. The MVD were $27.91 \pm 1.67$ (untreated) vs. $26.97 \pm 1.23$ (HPEI) vs. $26.74 \pm 0.85$ (HPEI+E-p) vs. 14.32 \pm 1.36 (HPEI+pVITRO-IP-10) $(\mathrm{p}<0.01$; Fig. 4B).

Antiangiogenesis activity was detected using the alginateencapsulated tumor cell assay; there were fewer newborn blood vessels in alginate beads from nude mice treated with HPEI+pVITRO-IP-10 than in those from the control groups. Moreover, compared with the three control groups, the levels of FITC-dextran uptake were significantly lower in mice treated with HPEI+pVITRO-IP-10. The FITC-dextran uptake were $5.62 \pm 0.46$ (untreated) vs. $5.49 \pm 0.45$ (HPEI) vs. 5.37 \pm 0.37 (HPEI+E-p) vs. $2.99 \pm 0.16 \mu \mathrm{g} /$ bead $($ HPEI+pVITRO-IP-10) $(\mathrm{p}<0.01$; Fig. 4C).

Induction of apoptosis in vivo. TUNEL assay was applied to test apoptotic cells in vivo. As described above, apoptotic cells were stained with bright green fluorescence, while normal live cells were stained by DAPI in bland blue. The TUNEL assay showed more apoptotic cells in the tumor sections of the HPEI+pVITRO-IP-10 groups, while there were few such cells in the three control groups. The apoptotic index, the ratio of bright green-fluorescence cells to total cells, revealed that tumors in the HPEI+pVITRO-IP-10 groups had significantly more TUNEL-positive nuclei than tumors in the untreated, HPEI, or HPEI+E-p groups. Apoptosis ratio was 5.79 \pm 1.67 (untreated) vs. $5.11 \pm 1.69$ (HPEI) vs. $8.38 \pm 2.16$ (HPEI+E-p) vs. 39.92 \pm 4.84 (HPEI+pVITRO-IP-10) as shown in Fig. 4D.

\section{Discussion}

A previous study showed that growth of xenografted human non-small cell lung cancer (NSCLC) can be suppressed by introducing IP-10 (7). IP-10 has been demonstrated to be a pleiotropic molecule eliciting potent biological effects, including stimulation of monocytes, natural killer (NK) cells and T-cell, regulation of T-cell and bone marrow progenitor maturation, modulation of adhesion molecule expression as well as inhibition of angiogenesis (6). However, the antitumoral efficacy of the IP-10 gene in human ovarian cancer is largely unknown. In the present study, we constructed a recombinant plasmid expressing IP-10 which was then used to effectively inhibit intraperitoneal xenograft growth of human ovarian cancer in nude mice, with biodegradable cationic HPEI nanogels as a novel delivery system. Treatment with HPEI+pVITRO-IP-10 complexes in nude mice was found to have no detectable toxicity.

Although the exact mechanism of pVITRO-IP-10 antitumor activity remains to be determined, part of the answer may lie in increased induction of antiangiogenesis and apoptosis. This assumption is strongly supported by the result of our experiments. Firstly, inhibition of angiogenesis was found in the tumors treated with HPEI+pVITRO-IP-10 compared with the control group. In this study, tube formation assay in vitro and CD31 staining, alginate-encapsulated tumor cell assay in vivo showed obvious inhibition of angiogenesis in HPEI+pVITRO-IP-10 treatment compared with controls. Secondly, more apoptotic cells were found in the tumors treated with HPEI+pVITRO-IP-10 than in control groups. In our study, the Hoechst/PI staining in vitro and the TUNEL assay in vivo suggested that $\mathrm{pVITRO}$ IP-10 transfected by HPEI resulted in significant inhibition of cell proliferation and induction of cell apoptosis. Other agents, including soluble vascular endothelial growth factor receptor (30), angiostatin (31), and endostatin $(32,33)$ have also been reported to inhibit tumor angiogenesis, induce apoptosis, thus suppress tumor growth. The molecular mechanisms underlying the inhibition of angiogenesis, and thus the induction of apoptosis in tumor cells, have yet to be elucidated fully (34). Taken together, these previous findings support our hypothesis that the antitumor efficacy in the present study may result in part from the inhibition of angiogenesis and induction of apoptosis. These proposed mechanisms are persuasive, but further experiments in vitro and in vivo are required to resolve the relative contribution of the hypothesized mechanism.

In ovarian cancer, which is mainly limited in the abdominal cavity, systemic administration usually could not attain desired drug concentration at the tumor site. Compared with systemic administration, intraperitoneal administration of HPEI+pVITRO-IP-10 avoids the systemic toxicity and first pass effect (35), refrains from clearance through bonding with plasma protein or phagocytized by lymphocyte that was inevitable in 
cationic lipid-DNA complexes, and maintains high focal concentration of the drug in the area where it was topically applied.

Consequently, we may wonder whether transferring the IP-10 gene into ovarian cancer cells by a suitable delivery system could be an approach for treating ovarian cancer. Over the last decade, many other functional genes have been reported to effectively inhibit ovarian cancer in vitro and in vivo $(36,37)$. However, the lack of safe and efficient gene delivery approach is a major obstacle to the applications of gene treatments in clinical practice. Currently, delivery systems for gene transfer are generally classified as viral and non-viral vectors. Non-viral vectors have many advantages over viral vectors, such as calcium phosphate, liposome, and emerging chitosan (38-43). In our study, a novel nonviral gene delivery system was developed based on PEI. In addition to the advantages of PEI, the delivery system resolved the transfection efficiency-dependent cytotoxicity and problems of non-biodegradability. The heparin conjugated PEI into biodegradable cationic nanogels were relatively stable in vitro and easy to degrade through enzymolysis and hydrolysis in vivo. As a natural polysaccharide, heparin is bio-compatible and non-toxic. Furthermore, it improves the biocompatibility of the nanogels. In our previous study, the transfection efficiency of HPEI was found to be comparable to that of PEI $25 \mathrm{k}$, but with less toxicity. Analyses of the erythrocyte aggregation and hemolysis showed that HPEI had better blood compatibility than PEI25K. Moreover, HPEI nanogels were stable in vitro and could be quickly degraded into the low-molecular weight chemical compound PEI and excreted in urine (15). We previously found that HPEI could effectively transfect plasmids into SKOV3 cells. Based on these advantages, HPEI nanogels were chosen to deliver a recombinant plasmid to evaluate the antitumor ability of IP-10, as well as the efficacy and safety of this delivery system.

In conclusion our study showed for the first time that the antitumor efficacy of a novel strategy of HPEI nanogeldelivered pVITRO-IP-10 could efficiently inhibit the growth of ovarian cancer both in vitro and in vivo. IP-10 as a potential angiogenesis inhibitor could efficiently inhibit the growth of xenografted ovarian cancer through inhibition of angiogenesis, inhibition of tumor cell proliferation and induction of tumorcell apoptosis. Biodegradable cationic HPEI nanogels might also be used as a safe and effective delivery media. Hence, HPEI nanogel-delivered pVITRO-IP-10 may have a great potential in clinical practice against ovarian cancer in the future. However, more studies are required to further demonstrate the mechanisms involved.

\section{Acknowledgements}

This study was supported by the National Key Basic Research Program (973 Program) of China (2010CB529905, 2011CB910700), National Natural Science Foundation of China (0040215401068), and NIH81071861.

\section{References}

1. Jermal A, Siegel R, Xu J and Ward E: Cancer statistics, 2010. CA Cancer J Clin 60: 277-300, 2010.

2. Jemal A, Siegel R, Ward E, Hao Y, Xu J and Thun MJ: Cancer statistics, 2009. CA Cancer J Clin 59: 225-249, 2009.

3. DiSaia PJ and Creasman WT: Epithelial ovarian cancer. In: Clinical Gynecologic Oncology. 5th edition, 1997.
4. Farber J and Mig M: IP-10: CXC chemokines that target lymphocytes. J Leukoc Biol 61: 246-247, 1997.

5. Loetscher M, Loetscher P, Brass N, Meese E and Moser B: Lymphocyte-specific chemokine receptor CXCR3: regulation, chemokine binding and gene localization. Eur J Immunol 28: 3696-3705, 1998.

6. Neville LF, Mathiak G and Bagasra O: The immunobiology of interferon-gamma inducible protein $10 \mathrm{kD}$ (IP-10): a novel, pleiotropic member of the CXC chemokine superfamily. Cytokine Growth Factor Rev 8: 207-219, 1997.

7. Douglas A, Arenberg A, Steven LK and Unkelfl PJ: Cisplatin-based adjuvant chemotherapy in patients with completely resected non-small-cell lung cancer. N Engl J Med 350: 351-360, 2004.

8. Maru SV, Holloway KA, Flynn G, Lancashire CL, Loughlin AJ, Male DK and Romero IA: Chemokine production and chemokine receptor expression by human glioma cells: role of CXCL10 in tumour cell proliferation. J Neuroimmunol 199: 35-45, 2008.

9. Fujita M,Zhu X,UedaR, SasakiK, Kohanbash G, KastenhuberER, McDonald HA, Gibson GA, Watkins SC and Muthuswamy R: Effective immunotherapy against murine gliomas using type 1 polarizing dendritic cells - significant roles of CXCL10. Cancer Res 69: 1587-1595, 2009.

10. Strieter RM, Polverini PJ, Kunkel SL, et al: The functional role of the ELR motif in CXC chemokine mediated angiogenesis. J Biol Chem 270: 348-357, 1995.

11. Boussif O, Lezoualc'h F, Zanta MA, et al: A versatile vector for gene and oligonucleotide transfer into cells in culture and in vivo: polyethylenimine. Proc Natl Acad Sci USA 92: 7297-7301, 1995.

12. Lungwitz U, Breunig M, Blunk T and Gopferich A: Polyethylenimine-based non-viral gene delivery systems. Eur J Pharm Biopharm 60: 247-266, 2005.

13. Neu M, Fischer D and Kissel T: Recent advances in rational gene transfer vector design based on poly (ethyleneimine) and its derivatives. J Gene Med 7: 992-1009, 2005.

14. Kunath K, Von HA, Fischer D, et al: Low-molecular weight polyethyleneimine as a non-viral vector for DNA delivery: comparison of physicochemical properties, transfection efficiency and in vivo distribution with high-molecular-weight polyethyleneimine. J Control Release 89: 113-125, 2003.

15. Gou M, Men K, Zhang J, et al: Efficient inhibition of C-26 colon carcinoma by VSVMP gene delivered by biodegradable cationic nanogel derived from polyethyleneimine. ACS Nano 4: 5573-5584, 2010.

16. Chuan X, Ma LG, Tao Y, et al: Efficient inhibition of ovarian cancer by truncation mutant of FILIP1L gene delivered by novel biodegradable cationic heparin-polyethyleneimine nanogels. Hum Gene Ther 22: 1413-1422, 2011.

17. Baudin B, Bruneel A, Bosselut N and Vaubourdolle M: A protocol for isolation and culture of human umbilical vein endothelial cells. Nat Protoc 2: 481-485, 2007.

18. Wen Y, Wang C, Ma T, Li Z, Zhou L, Mu B, Leng F, Shi H, Li Y and Wei Y: Immunotherapy targeting fibroblast activation protein inhibits tumor growth and increases survival in a murine colon cancer model. Cancer Sci 101: 2325-2332, 2010.

19. Qiaoyun S, Andrew T, Guyen N, Angell Y, et al: A combinatorial approach for targeted delivery using small molecules and reversible masking to bypass non-specific uptake in vivo. Gene Ther 17: 1085-1097, 2010.

20. Jen-Tsan C, Howard YC, Nancy NW, Dustin SC, Nina D and Patrick OB: Genome wide view of gene silencing by small interfering RNAs. Proc Natl Acad Sci USA 11: 6343-6346, 2003.

21. Selvakumaran M,Pisarcik DA, Bao R, Yeung AT and Hamilton TC: Enhanced cisplatin cytotoxicity by disturbing the nucleotide excision repair pathway in ovarian cancer cell lines. Cancer Res 63: 1311-1316, 2003.

22. Xu GX, Zhong Y, Munir S, Yang BB, Tsang BK and Peng C: Nodal induces apoptosis and inhibits proliferation in human epithelial ovarian cancer cells via activin receptor-like kinase 7. J Clin Endocrinol Metab 89: 5523-5534, 2004.

23. Lin XJ, Chen XC, Wei YQ, et al: Efficient inhibition of intraperitoneal human ovarian cancer growth and prolonged survival by gene transfer of vesicular stomatitis virus matrix protein in nude mice. Gynecol Oncol 104: 540-546, 2007.

24. Folkman J: Angiogenesis: an organizing principle for drug discovery. Nat Rev Drug Discov 6: 273-286, 2007.

25. Hillen $F$ and Griffioen AW: Tumour vascularization: sprouting angiogenesis and beyond. Cancer Metastasis Rev 26: 489-502, 2007. 
26. Lin XJ, Chen XC, Wang L, et al: Dynamic progression of an intraperitoneal xenograft model of human ovarian cancer and its potential for preclinical trials. J Exp Clin Cancer Res 26 : 467-474, 2007.

27. Sun C, Yi T, Song X, Li S, Qi X, Chen X, Lin H, He X, Li Z, Wei Y and Zhao X: Efficient inhibition of ovarian cancer by short hairpin RNA targeting claudin-3. Oncol Rep 26: 193-200, 2011.

28. Hoffmann J, Schirner M, Menrad A and Schneider MR: A highly sensitive model for quantification of in vivo tumor angiogenesis induced by alginate-encapsulated tumor cells. Cancer Res 57: 3847-3851, 1997.

29. Fan Y, Li Z, Hong XD, et al: Efficient inhibition of ovarian cancer growth and prolonged survival by transfection with a novel pro-apoptotic gene, hPNAS-4, in a mouse model. Oncology 75 137-144, 2008 .

30. Takayama K, Ueno H, Nakanishi Y, et al: Suppression of tumor angiogenesis and growth by gene transfer of a soluble form of vascular endothelial growth factor receptor into a remote organ. Cancer Res 60: 2169-2177, 2000.

31. Yokoyama Y, Dhanabal M, Griffioen AW, Sukhatme VP and Ramakrishnan S: Synergy between angiostatin and endostatin: inhibition of ovarian cancer growth. Cancer Res 60: 2190-2196, 2000.

32. Herbst RS, Mullani NA, Davis DW, et al: Development of biologic markers of response and assessment of antiangiogenic activity in a clinical trial of human recombinant endostatin. J Clin Oncol 20: 3804-3814, 2002

33. Schmidt NO, Ziu M, Carrabba G, et al: Antiangiogenic therapy by local intracerebral microinfusion improves treatment efficiency and survival in an orthotopic human glioblastoma model. Clin Cancer Res 10: 1255-1262, 2004.
34. Folkman J: Angiogenesis and apoptosis. Semin Cancer Biol 13: 159-167, 2003.

35. Alberts DS, Clouser MC, Hess LM, Springer V, Heidel B and Howell SB: Selection of drugs for intraperitoneal chemotherapy for ovarian cancer. Intraperitoneal Ther Ovarian Cancer 1: 77-88, 2010.

36. Malek A, Bakhidze E, Noske A, et al: HMGA2 gene is a promising target for ovarian cancer silencing therapy. Int $\mathrm{J}$ Cancer 123 : 348-356, 2008

37. Nguyen TMB, Subramanian IV, Xiao X, et al: Adenoassociated virus-mediated delivery of kringle 5 of human plasminogen inhibits orthotopic growth of ovarian cancer. Gene Ther 17: 606-615, 2010

38. Young LS and Mautner V: The promise and potential hazards of adenovirus gene therapy. Gut 48: 733-736, 2001.

39. Zhdanov RI, Podobed OV and Vlassov V: Cationic lipid-DNA complexes - lipoplexes - for gene transfer and therapy. Bioelectrochemistry 58: 53-64, 2002.

40. Gao Y, Xu Z, Chen S, et al: Arginine-chitosan/DNA selfassemble nanoparticles for gene delivery: in vitro characteristics and transfection efficiency. Int J Pharm 359: 241-246, 2008.

41. Montier T, Benvegnu T, Jaffre's PA, et al: Progress in cationic lipid-mediated gene transfection: a series of bioinspired lipids as an example. Curr Gene Ther 8: 296-312, 2008.

42. Morille M, Passirani C, Vonarbourg A, et al: Progress in developing cationic vectors for non-viral systemic gene therapy against cancer. Biomaterials 29: 3477-3496, 2008.

43. Suzuki R, Namai E, Oda Y, et al: Cancer gene therapy by IL-12 gene delivery using liposomal bubbles and tumoral ultrasound exposure. J Control Release 142: 245-250, 2010. 